\title{
Posterior tibial slope measurement among Saudi population
}

\begin{abstract}
To measure the posterior slope of the tibia among the healthy Saudi population using Magnetic Resonance Imaging (MRI). A retrospective study review of 151 knee MRIs at King Khaled University Hospital, Riyadh, Saudi Arabia. All patients with no previous surgical intervention to their knees and did not suffer any bone injury around the knee were included. Three different orthopedic physicians (two senior residents, one orthopedic fellow) measured the posterior tibial slope (PTS) angle for all patients, and their average was taken for all readings using a sagittal T2 MRI cut. Patients with a history of previous surgical intervention to the knee joint, trauma involving distal femur or proximal tibia, osteoarthritis and inflammatory arthritis, and congenital deformities were excluded from the study. The mean age of patients was 28.15 in a range of $15-50$ years. The posterior tibial slope mean and the median and the mode were $8.76,8.73,7.53$, respectively. In addition, the mean angle (degree) in females was $9.69 \pm 4.02$ and $8.76 \pm 4.15$ in males. The Maximum and the minimum posterior slope angle calculated in these patients were respectively 19.73 and 0.3 degrees. Our findings are that the mean posterior tibia slope among the Saudi population is $8.76^{\circ}$. The results showed the difference of PTS in Saudi people comparing to deferent communities. The association between the numbers of the posterior tibial slope with gender was tested and no significant correlation. In this study, we did not calculate weight and height in the cases as independent factors. Because of that, upcoming studies might consider these factors.
\end{abstract}

Keywords: posterior tibial slope, MRI, arthroplasty, tibia

Volume I3 Issue 3 - 202 I

\author{
Mouad Alsowaigh,' Mohammad A Arafah, ${ }^{2}$ \\ Sultan Khaled Alharbi, ${ }^{3}$ Omar AlSultan, ${ }^{4}$ Adel \\ Alshahrani, ${ }^{5}$ Fahad $\mathrm{H} \mathrm{Al} \mathrm{Hulaibi,}{ }^{6}$ Ahmad bin \\ Nasser ${ }^{7}$ \\ 'King Saud university fellow sponsored by King Fahad Hospital \\ Hofuf, King Saud University, Saudi Arabia \\ ${ }^{2}$ Ministry of health, Orthopedic surgeon, Saudi Arabia \\ ${ }^{3}$ Department of Orthopeadic Surgery, Majmaah University, Saudi \\ Arabia \\ ${ }^{4}$ Orthopedic surgeon, King Saud University, Saudi Arabia \\ ${ }^{5}$ Associate Professor, Physical therapy, Najran University, Saudi \\ Arabia \\ ${ }^{6}$ Orthopedic Surgery, Dammam Medical complex, Saudi Arabia \\ ${ }^{7}$ Assistant Professor, orthopedic Surgery Department, College \\ of Medicine, King Saud University, Saudi Arabia
}

Correspondence: Adel Alshahrani, Associate Professor, Physical therapy, Najran University, Saudi Arabia, Tel+9665482260 I I, Email amsalshahrani@nu.edu.sa

Received: May 26, 2021 | Published: June 18, 2021
Abbreviations: PTS, posterior tibial slope; MRI, magnetic resonance imaging

\section{Introduction}

Maintaining long limb axis and biomechanics is often the cornerstone of orthopedic surgeries. ${ }^{1}$ Many studies have attributed failures and decreased longevity of some surgeries to the disruption of alignment, the axis of lower limbs. The posterior tibial slope is an important parameter to consider in total knee arthroplasty and sportrelated reconstructive procedures, e.g., anterior and posterior cruciate ligament reconstruction. ${ }^{2}$ It is defined as the slope of the medial tibial plateau relative to the longitudinal axis on the sagittal plane. ${ }^{3}$ Stability and posterior cruciate ligament tension were affected by the degrees of the posterior slope of the tibia.

Moreover, several studies have concluded that increased tibial slope significantly correlates with increased posterior tibial translation. ${ }^{4}$ Increase PTS is known to be a risk factor for the rapture of anterior cruciate ligament (ACL) in native knees. ${ }^{5,6}$ Where decrease PTS angel as a risk factor for $r$ posterior cruciate ligament (PCL) injury. ${ }^{7}$

This study aims to measure the posterior slope of the tibia among the healthy Saudi population using Magnetic resonance imaging (MRI) obtained for various indications. To the best of our knowledge, no previous study focused on Posterior tibial slope (PTS) measurement among the Saudi population.

\section{Materials and methods}

A retrospective review of 151 knee MRIs (103 males, 48 females) at King Saud University medical city Hospital, Riyadh, Saudi Arabia, was done. The IRB approval was taken with project number E-205155 from health sciences colleges' research on human subjects. The patients' ages ranged between 16-47 years old. Those patients were seen before in the clinic due to knee pain and MRIs were requested as needed. After reviewing the patients' files, all included patients in our study had no previous surgical intervention to their knees and did not suffer any bone injury around the knee. Three different orthopedic physicians (two senior residents, one orthopedic fellow) measured the PTS angle for all patients and their average was taken for all readings.

A sagittal T2 MRI cut in the middle of the medial tibial compartment was chosen for all patients to measure the PTS angle. First, the PTS angle was measured by drawing an intramedullary line parallel to the volar cortex of the tibia, and a perpendicular line is drawn over our first line. Then, a third line to be drawn between the highest bony tips of the anterior and posterior aspect of the tibial plateau. Finally, the second and third line is measured angle delineated the posterior tibial slope (Figure $1 \& 2$ ).

Exclusion criteria included patients with a history of previous surgical intervention to the knee joint, trauma involving distal femur or proximal tibia, osteoarthritis or inflammatory arthritis, and congenital deformities.

\section{Statistical analysis}

The collected data were analyzed using (SPSS 22; IBM Corp., New York, NY, USA). Continuous variables in the study were translated as mean \pm standard deviation. Cronbach's Alpha was chosen to assess the reliability of measuring the posterior tibial slope. A p-value $<0.05$ was in our study considered statistically significant.

\section{Reliability of measuring}

The Cronbach's alpha value was 0.99 , which reflects the excellent reliability of the measuring. 


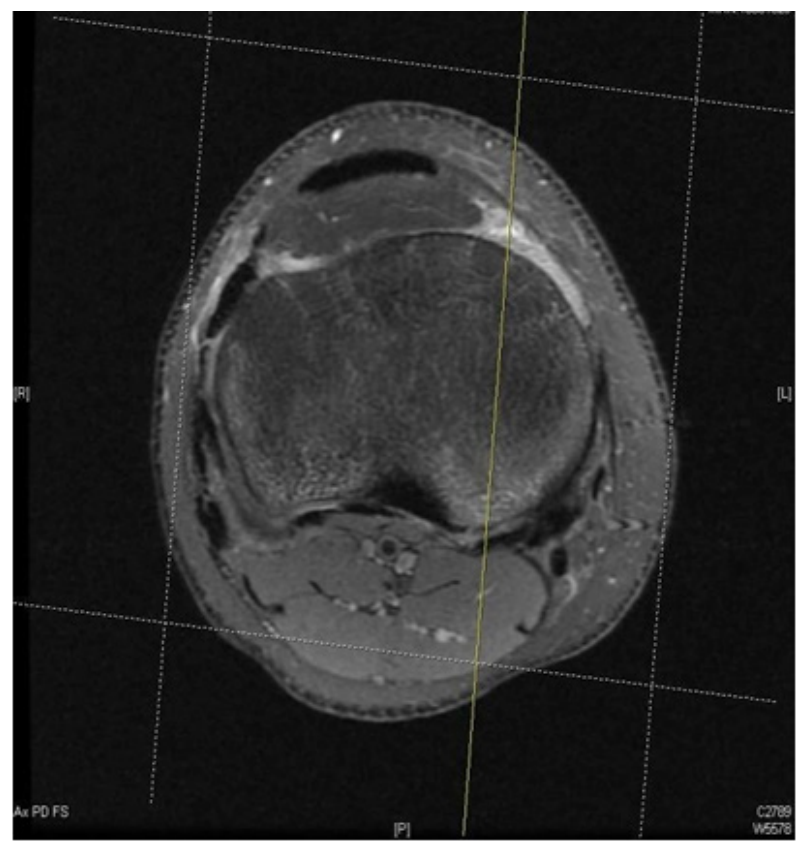

Figure I Chosen cut for middle compartment.

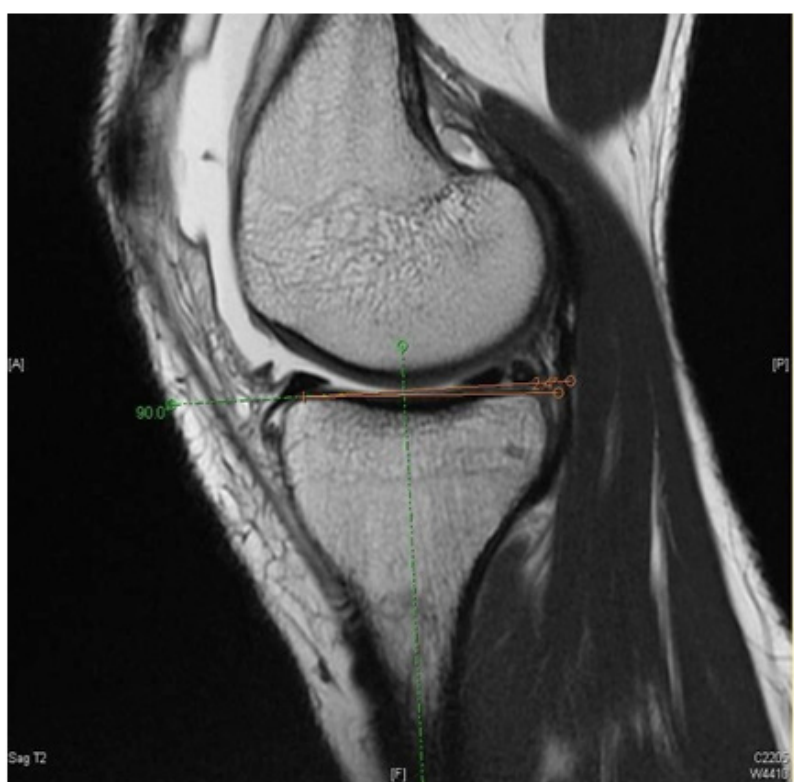

Figure 2 Technique of measuring the PTS.

\section{Results}

One hundred and fifty-one knee MRIs were reviewed by two senior residents and one orthopedic fellow in this study. Our patients' ages ranged from 15 to 50 years old with a mean of $28.15 \pm 7.81$, as described in Figure 3. The maximum difference between the readings of each reviewer was less than 2 degrees for all patients, and the average results for reviewers were used to analyze our findings. The mean posterior tibial slope was $9.06 \pm 4.12$ degrees. When comparing the PTS angle between males and females, the mean PTS angles were $8.76 \pm 4.15$ and $9.69 \pm 4.02$, respectively (Table $1 \& 2$ ). Thus, the maximum reported PTS angle was 19.73 degrees, and the minimum angle was 0.3 degrees. Figure 3 illustrates the percentage of different PTS angles for each sex. Figure 4 shows the number of patients with PTS angles higher or lower than 10 degrees.

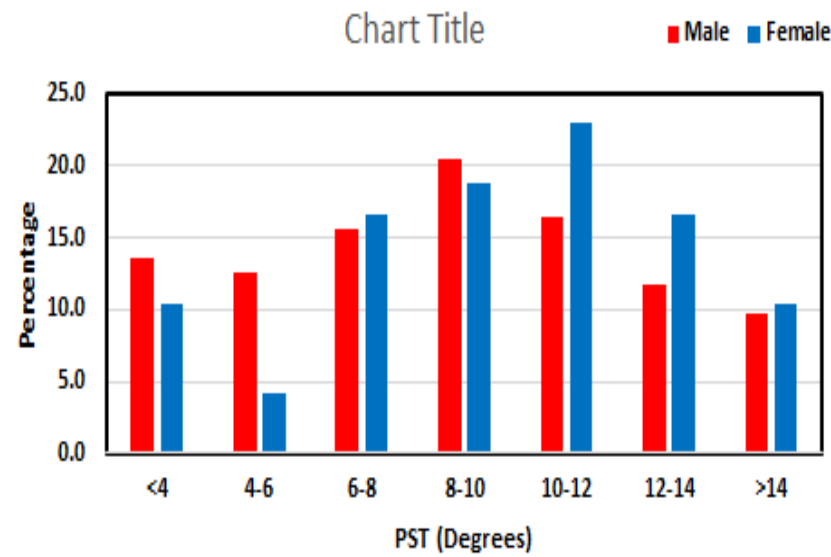

Figure 3 Percentage (\%) of different tibial plateau slope for both gender.

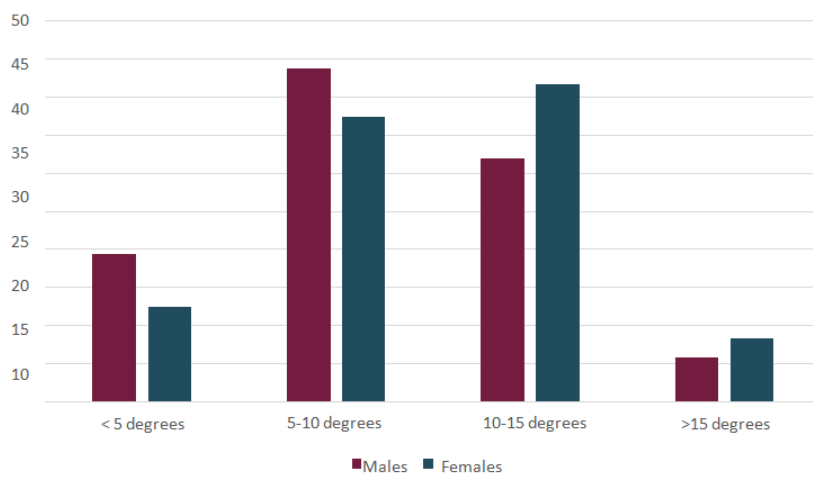

Figure 4 Percentage of different PTS angles for both sexes.

Table I Characteristics of the patients

\begin{tabular}{llll}
\hline & & $\begin{array}{l}\text { Number of knees } \\
(\mathbf{n}=15 \text { I })\end{array}$ & $\%$ \\
\hline Gender & Male & 103 & 68.2 \\
& Female & 48 & 31.8 \\
Side & Right & 86 & 57 \\
& Left & 65 & 43 \\
Age( Mean \pm SD $)$ & & 28.15 & 7.81
\end{tabular}

Table 2 Number of study subjects in terms of tibial plateau slope with less or more than 10 degrees

\begin{tabular}{|c|c|c|c|c|c|c|}
\hline & & \multicolumn{2}{|c|}{ Slope < I0 } & \multicolumn{3}{|l|}{ Slope $\geq 10$} \\
\hline & & Number & $\%$ & Number & $\%$ & $P$ value \\
\hline All & & 85 & 56.3 & 66 & 43.7 & \\
\hline \multirow[t]{2}{*}{ Gender } & Male & 63 & 61.2 & 40 & 38.8 & 0.077 \\
\hline & Female & 22 & 45.8 & 26 & 54.2 & \\
\hline \multirow[t]{2}{*}{ Side } & Right & 46 & 53.5 & 40 & 46.5 & 0.424 \\
\hline & Left & 39 & 60 & 26 & 40 & \\
\hline
\end{tabular}




\section{Discussion}

The standard range for the posterior tibial plateau slope has not been fully reported out for all populations. The impact of determining the angle and direction will have a major scientifically and economically. The increasing numbers of knee surgical interventions and the significant role to achieve acceptable lower limb biomechanics.

Measurement of PTS was discussed controversially. Previously, the tibial slop was guided by a lateral view X-ray of the knee. However, it has some limitations in accuracy in rotation wise and overlap between medial and lateral tibia plateau. ${ }^{13}$ Then CT scan came in line with 3D-level with measurement of lateral and the medial articular surfaces of the proximal tibia containing plateau and rim..$^{14,15}$ Furthermore, the MRI technique is evolving new protocols. Both CT scan and MRI were equal to the manual measurements and showed high reliability in measuring the tibial slope. CT and MRI also eliminate rotating errors by reconstruction and 3D imaging, giving an exact lateral view. Both are equal to evaluate the same PTS. ${ }^{13}$

In our study, the slope range we have found was $0.3^{\circ}-19.73^{\circ}$, averaging $9.06^{\circ}$. Our female population slope was higher than male (mean of 9.69 vs 8.76). This is similar to Hashemi et al., ${ }^{1}$ whose results were females higher than males.

Moore et al. ${ }^{2}$ published the slope number with a range of $14 \pm 3.7$ degree (7-22 degree) in a sample of 50 American. Matsuda reported lower numbers around 10.7 degrees (5-15.5 degree) in a sample of 30 Japanese, ${ }^{10}$ and Chiu et. reported the average slope range of $14.7 \pm 3.7^{\circ}$ $\left(5-22^{\circ}\right)$ in a sample of 25 Chinese, ${ }^{16}$ on the other hand, our study, the association between the numbers of posterior tibial slope in relation to gender were tested. A significant correlation was not found among PTS and these two factors with a p-value more than $0.05(\mathrm{P} \geq 0.05)$. The numbers are quite similar with different studies was done in Iran. ${ }^{3}$ In addition, the PTS of more than 10 and less than 10 were compared with both sides (right and left), with no significant correlation were found $(\mathrm{P} \geq 0.05)$. Aljuhani et al. tested the PTS among Saudi people using plain radiographs, and the average physiological posterior tibia slope angle was $13.6 \pm 3.4^{\circ}$ (range: $3.8-23.9^{\circ}$ ). There was no correlation between age, gender and PTS angles. ${ }^{18}$

The values showed the difference of PTS in Saudi people comparing to deferent communities. Therefore, more comprehensive studies to search for the reported difference and calculation of actual PTS number of the Saudi community are needed. These differences could be due to multiple reasons such as race and genetic factors.... etc. In addition, Saudi's lifestyle is unique to other nations in term of movements in prayer and sitting on the floor, could be considered as effective factors.

Given the large area of distribution in Saudi Arabia and the significance of morphological and anatomical indicators, our study suggests carrying out widely similar studies in other areas. In this study, we did not calculate weight and height in the cases as independent factors. Because of that, upcoming studies might consider these factors.

\section{Conclusion}

The results showed a difference in posterior tibia slope in the Saudi population compared to deferent communities. The Average posterior tibia slope in the Saudi population is 9.06. Furthermore, no significant difference between the degree of the posterior tibial slope in relation to gender, with a P-value of $>0.05$. Upcoming studies might consider calculating weight and height in the cases as independent factors with a larger sample size.

\section{Author contributions}

In this research project, conceptualization, Mouad Alsowaigh, and Ahmed Bin Nasser; methodology, Mohammed Arafa, Sultan Alharbi, and Omar Alsultan; validation, Mouad Alsowaigh, Ahmed Bin Nasser, Mohammed Arafa, Sultan Alharbi,and Omar Alsultan; formal analysis, Mouad Alsowaigh; investigation, Mouad Alsowaigh,Ahmed Bin Nasser, Mohammed Arafa, Sultan Alharbi, and Omar Alsultan; resources, Ahmed Bin Nasser; data curation, Ahmed Bin Nasser, and Adel Alshahrani; writing-original draft preparation, Mouad Alsowaigh, and Ahmed Bin Nasser; writing-review and editing, Adel Alshahrani Fahad AL Hulaibi; visualization, Fahad Al Hulaibi; supervision, Ahmed Bin Nasser; project administration, Mouad Alsowaigh.. All authors have read and agreed to the published version of the manuscript.

\section{Institutional review board statement}

The study was conducted according to the guidelines of the Declaration of Helsinki and approved by the Institutional Review Board of King Saud University (protocol code E-20-5155 and date of approval 20 Oct 2020).

Informed Consent Statement: Informed consent was obtained from all subjects involved in the study.

\section{Acknowledgments}

None.

\section{Conflicts of interest}

The authors declare no conflict of interest.

\section{References}

1. Javad Hashemi, Naveen Chandrashekar, Brian Gill, et al. The geometry of the tibial plateau and its influence on the biomechanics of the tibiofemoral joint. J Bone Joint Surg. 2008;90(12):2724-2734.

2. Moore TM, Harvey JP. Jr Roentgenographic measurement of tibial- plateau depression due to fracture. J Bone Joint Surg Am. 1974;56(1):155-160.

3. JM Muthuuri. Determination of posterior tibia slope and slope deterioration with osteoarthritis: a radiological study in an african population. EAOJ. 2014;8(1).

4. Mohamad Qoreishi M, Syavash Hemmati M, Ali Sina Shahi M, et al. Measurement of posterior tibial slope (a cross-sectional study in Tehran). JBS J. 2015;2(1):10.

5. Griffin LY, Albohm MJ, Arendt EA, et al. Understanding and preventing noncontact anterior cruciate ligament injuries: a review of the Hunt Valley II meeting, January 2005. Am J Sports Med. 2006;34(9):1512-1532.

6. Brandon ML, Haynes PT, Bonamo JR, et al. The association between posterior-inferior tibial slope and anterior cruciate ligament insufficiency. Arthroscopy. 2006;22(8):894-899.

7. Bernhardson AS, DePhillipo NN, Daney BT, et al. Posterior Tibial slope and risk of posterior cruciate ligament injury. Am J Sports Med. 2019;47(2):312-317.

8. Genin P, Weill G, Julliard RJ. The tibial slope. Proposal for a measurement method. Radiol. 1993;74(1):27-33.

9. Bernhardson AS, dephillipo NN, Daney BT, et al. Posterior Tibial Slope and Risk of Posterior Cruciate Ligament Injury. Am J Sports Med. 2019;47(2):312-317.

10. Schatka I, Weiler A, Jung TM, et al. High tibial slope correlates with increased posterior tibial translation in healthy knees. Knee Surg Sports Traumatol Arthrosc. 2018;26(9):2697-2703. 
11. Karimi E, Norouzian M, Birjandinejad A, et al. Measurement of Posterior Tibial Slope Using Magnetic Resonance Imaging. The archives of bone and joint surgery. 2017;5(6):435-439.

12. Kacmaz IE, Topkaya Y, Basa CD, et al. Posterior tibial slope of the knee measured on X-rays in a Turkish population. Surg Radiol Anat. 2020;42(6):673-679.

13. Utzschneider S, Goettinger $\mathrm{M}$, Weber $\mathrm{P}$, et al. Development and validation of a new method for the radiologic measurement of the tibial slope. Knee Surg Sports Traumatol Arthrosc. 2011;19(10):1643-1648.

14. Zhang $\mathrm{K}$, Han Q, Wang $\mathrm{H}$, et al. Measurement of proximal tibial morphology in northeast Chinese population based on threedimensional reconstruction computer tomography. Medicine (Baltimore). 2019;98(45):e17508.
15. Hoch A, Jud L, Roth $\mathrm{T}$, et al. A real $3 \mathrm{D}$ measurement technique for the tibial slope: differentiation between different articular surfaces and comparison to radiographic slope measurement. BMC Musculoskelet Disord. 2020;21(1):635.

16. Tang WM, Zhu YH, Chiu KY. Axial alignment of the lower extremity in Chinese adults. J Bone Joint Surg Am. 2000;82(11):1603-1608.

17. Hsu RW, Himeno S, Coventry MB, et al. Normal axial alignment of the lower extremity and load-bearing distribution at the knee. Clin Orthop Relat Res. 1990;255(1):215-227.

18. ALJuhani W, Qasim SS, Alsalman M. Variability of the Posterior Tibial Slope in Saudis: A Radiographic Study. Cureus. 2020;12(9):e10699. 